\title{
Spectral variability in the Seyfert 2 galaxy NGC 4388
}

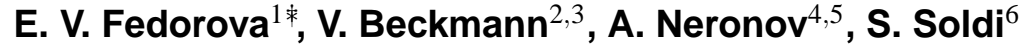 \\ ${ }^{1}$ Astronomical Observatory of the Kiev Taras Schevchenko University, Kiev 03040, Ukraine \\ ${ }^{2}$ François Arago Centre, APC, Université Paris Diderot, CNRS/IN2P3, CEA/DSM, Observatoire \\ de Paris, 13 rue Watt, 75205 Paris Cedex 13, France \\ ${ }^{3}$ CSST, University of Maryland Baltimore County, 1000 Hilltop Circle, Baltimore, MD 21250, \\ USA \\ ${ }^{4}$ ISDC Data Centre for Astrophysics, Chemin d'Écogia 16, 1290 Versoix, Switzerland \\ ${ }^{5}$ Observatoire Astronomique de l'Université de Genève, Ch. Des Maillettes 51, 1290 Sauverny, \\ Switzerland \\ ${ }^{6}$ Laboratoire AIM - CNRS - CEA/DSM - Université Paris Diderot (UMR 7158), CEA Saclay, \\ DSM/IRFU/SAp, 91191 Gif-sur-Yvette, France
}

The analysis of INTEGRAL IBIS/ISGRI and JEM-X data obtained during six years (from 2003 to 2009) of the Seyfert 2 galaxy NGC 4388 indicates that both X-ray flux and the shape of the broadband 3-300 keV spectrum are variable. The averaged X-ray to soft $\gamma$-ray continuum emission of NGC 4388 is well represented by an absorbed cut-off power-law model, with photon index $\Gamma=1.68 \pm 0.04$ and a cut-off energy of $E_{C}=204_{-32}^{+44} \mathrm{keV}$. The flux in the $20-60 \mathrm{keV}$ energy range and the spectral shape vary on timescales of months. During the first low-flux phase the cut-off appears to be near $80 \mathrm{keV}$, while it moves slightly, within the error limits, up to 110 $\mathrm{keV}$ when the flux is high. In a second low-flux phase no cut-off is detectable, so we can see here at least three different cases depending on the spectral shape and flux level: dim and bright phases with hard spectrum and high-energy cut-off as well as dim, soft spectrum phase with no cut-off. We compare the observed flux states with those detected in other Seyfert galaxies.

8th INTEGRAL Workshop "The Restless Gamma-ray Universe"

September 27-30 2010

Dublin Castle, Dublin, Ireland

\footnotetext{
*E-mail:efedorova@ukr.net

† Speaker.
} 


\section{Introduction}

NGC 4388 is the brightest Seyfert 2 galaxy in the X-rays with a redshift of $z=0.0084$ [1]. Its X-ray emission is absorbed by a medium with variable column density in the range $10^{23} \mathrm{~cm}^{-2}<\mathrm{N}_{\mathrm{H}}<10^{24} \mathrm{~cm}^{-2}[7,11]$. The first X-ray observation of NGC 4388 was done by SL2-XRT, which measured an absorbtion of $N_{\mathrm{H}} \simeq 10^{23} \mathrm{~cm}^{-2}$ [11]. The results of XMM-Newton and $R X T E$ observations have shown that the absorbing medium density is strongly variable [7].

BeppoSAX observed the first signs of a high-energy cut-off above $100 \mathrm{keV}$ [20]. Later the Swift/BAT All-Sky Survey detected slow flux variability in the energy range 14-195 keV [2]. The latest observations by Suzaku [8] showed fast (hour-scale) variations of the direct nuclear component of NGC 4388.

Here we present the results of our treatment of the INTEGRAL data on NGC 4388 obtained during six years of observations, i.e. a larger data set than considered in previous works $[1,3]$. The longer exposure time enables us to achieve better statistics and to detect the cut-off at high energies. We also investigate its long-term variability. In Section 2 we describe the data reduction and the spectral analysis. Combining the data from different instruments on-board INTEGRAL we obtain the spectrum of the source in the 3-300 keV energy range. In Section 3 we study the variability of the AGN. Finally, in Section 4 we discuss our results and compare them with other $\mathrm{X}$-ray observations.

\section{Data analysis}

The data analyzed here include all publicly available INTEGRAL data on the source as of July 2009, i.e. 1344 science windows (SCW) from spacecraft revolutions up to 762 for the IBIS/ISGRI instrument. For SPI and both JEM-X detectors there were 586 and 154 SCWs, respectively. The total exposure time of the data set used for the IBIS/ISGRI lightcurves is $\sim 4.2 \mathrm{Ms}$, including all observations when NGC 4388 was within $15^{\circ}$ off-axis angle. To obtain the spectra we have used a smaller data set (off-axis angle $<10^{\circ}$ ) of $840 \mathrm{ScWs}$ (total exposure $\sim 2.1 \mathrm{Ms}$ ). For SPI $\sim 1.5 \mathrm{Ms}$ of data are available (off-axis angle $<10^{\circ}$ ), and both JEM-X detectors accumulated a total exposure time of $\sim 500 \mathrm{ks}$ (off-axis angle $<3^{\circ}$ ). ISGRI and SPI data analysis was performed using standard procedures of OSA 7.0. For the JEM-X data we used OSA 9, extracting the spectra from combined JEM-X1 and JEM-X2 mosaic images.

Spectral modelling was done using the XSPEC 12 package. For the analysis we considered the following set of models (see e.g. [20] for a physical motivation of this choice of models): an absorbed power-law model $i$ (zwabs.powerlaw model of XSPEC); an absorbed power-law with a high-energy cut-off $i i$ (zwabs-cutoffpowerlaw); an absorbed cut-off power-law plus Compton-reflection component iii (zwabs-pexrav [15]) which describes the reflection of the primary AGN emission on either the accretion disk, a hot corona, or the cold absorbing gas surrounding it; an absorbed power-law with a Compton-reflection component $i v$ (zwabs.reflect, [15]) but without cut-off at high energies. We did not include the Fe K $\alpha$ line seen e.g. in XMMNewton data [1,7] when fitting the INTEGRAL data because the spectral resolution of the JEM-X did not allow a significant detection. Due to different exposure times we have included intercalibration factors for JEM-X $\left(\mathrm{K}_{X}\right)$ and SPI $\left(\mathrm{K}_{\gamma}\right)$ spectra. 


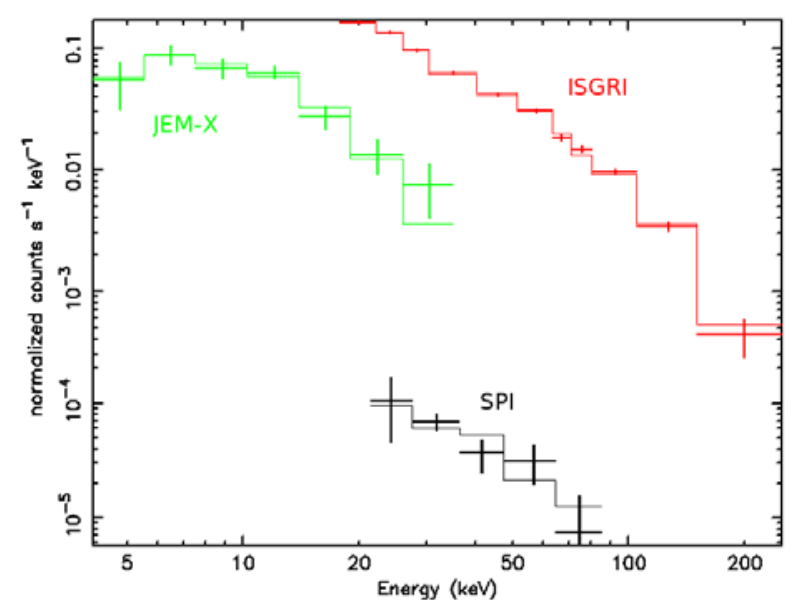

Figure 1: Folded full exposure time INTEGRAL spectra.

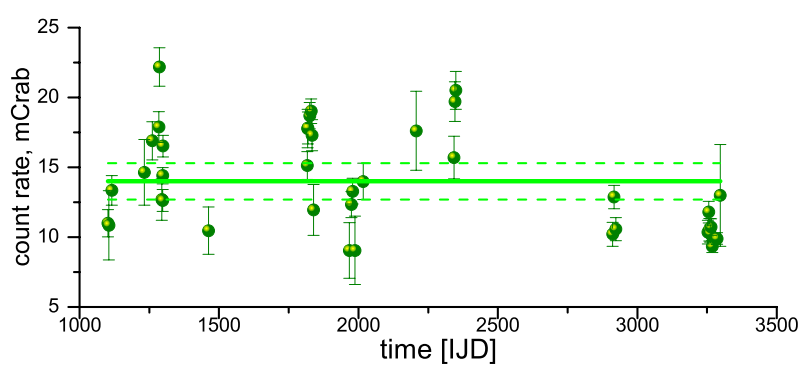

Figure 2: IBIS/ISGRI 20-60 keV lightcurve. The mean values of the count rate and errors are shown by the solid and dashed lines. Time is given in INTEGRAL Julian Date (IJD = MJD -51,544.0).

The results of the fit to the overall spectra demonstrate that the observed spectrum is inconsistent with a simple absorbed power-law model. The fit significantly improves when a Comptonreflection model is applied (F-test probability is 0.07), but a model with high-energy cut-off gives a still better representation than a reflected one (F-test probability is 0.009). Model iii is unreasonable compared to model ii. Thus the absorbed power-law model with high-energy cut-off is the best-fit for the INTEGRAL spectrum based on all available data. It provides $\chi^{2} /$ d.o.f $=19.4 / 18$ and a photon index $\Gamma=1.68 \pm 0.04$, a cut-off energy $E_{\mathrm{C}}=209_{-32}^{+44} \mathrm{keV}$, and a column density $N_{\mathrm{H}}=4.0_{-1.0}^{+1.2} \mathrm{~cm}^{-2}$ consistent with the one found in ASCA and BeppoSAX observations [7], i.e. near $4 \times 10^{23} \mathrm{~cm}^{-2}$. Unabsorbed model fluxes are: $(1.52 \pm 0.09) \times 10^{-10} \mathrm{erg} \mathrm{cm}^{-2} \mathrm{~s}^{-1}$ in the $4-20$ keV band, $(1.41 \pm 0.02) \times 10^{-10} \mathrm{erg} \mathrm{cm}^{-2} \mathrm{~s}^{-1}$ at $20-60 \mathrm{keV}$, and $(1.56 \pm 0.02) \times 10^{-10} \mathrm{erg} \mathrm{cm}^{-2} \mathrm{~s}^{-1}$ in the 60-200 keV band. The intercalibration factors are $\mathrm{K}_{X}=1.02 \pm 0.13$ and $\mathrm{K}_{\gamma}=0.71 \pm 0.15$. Folded INTEGRAL spectra are shown in Fig.1.

\section{Variability analysis}

To study the variability of NGC 4388 we extracted the lightcurve from INTEGRAL/ISGRI data in the 20-60 keV energy band (shown in Fig. 2). Time bins in this lightcurve are from 1 to 5 


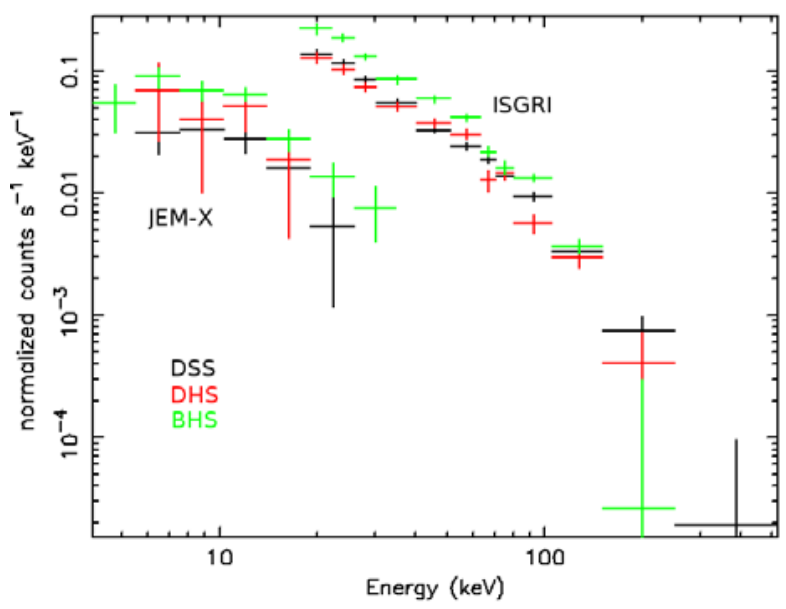

Figure 3: The folded spectra during the BHS, DSS and DHS phases (JEM-X and ISGRI).

days, depending on the exposure times of observations in separate revolutions. As it can be seen in the lightcurve, we can divide the observations into intervals of flux higher and lower than the mean value of $14 \mathrm{mCrab}$ in the $20-60 \mathrm{keV}$ band. Spectral modelling during each revolution shows that there are at least three different spectral states depending on both the $20-60 \mathrm{keV}$ flux level and the hardness ratio $\mathrm{HR}=\frac{F_{>60 \mathrm{keV}}-F_{20-60 \mathrm{keV}}}{F_{>60 \mathrm{keV}}+F_{20-60 \mathrm{kev}}}$, where $F_{>60 \mathrm{keV}}$ is the flux above $60 \mathrm{keV}$ (the upper boundary is varying from 200 to $500 \mathrm{keV}$ depending on the spectral state). We define hereafter the "bright, hard spectrum" phase (BHS) as characterized by the highest flux level ( $>14 \mathrm{mCrab}$ ) and a hardness ratio of $H R<0.2$. In the "dim, hard spectrum" phase (DHS) the source has $<14 \mathrm{mCrab}$ and has a similar hardness ratio as during the BHS phase. In addition, we define an "dim, soft spectrum" phase (DSS) with low fluxes and an hardness ratio of $H R>0.2$, i.e. softer than during the BHS and DHS phases. To trace out how the changes in the source flux correspond to changes in the spectrum, we split the data into three parts corresponding to the three phases and compare the spectra. BHS, DHS and DSS spectra are shown in Fig. 3 and the corresponding best-fit parameters are given in Table 1. For both BHS and DHS spectra, following the results of an F-test analysis, the best-fit model is $i i$, the absorbed cut-off power law model. The $i$ model is obviously insufficient, and also iii results in bad $\chi_{v}^{2}$ values. Note that the cut-offs detected during BHS and DHS phases overlap within the error limits, despite the significant difference in flux level. At the same time during the DSS phase no cut-off can be seen in the spectrum (all the models more complicate than $i$ give worse fitting results), simultaneously with almost the same flux level as during the DHS one. This lead us to the conclusion that the flux level and spectral shape in NGC 4388 are not correlated.

\section{Discussion}

We have analyzed the INTEGRAL data collected over six years to study the flux and spectral variability in the Seyfert 2 galaxy NGC 4388. We derive a maximum flux change by a factor of $\sim 2$ in the $20-60 \mathrm{keV}$ band on time scales of $1-2$ months, consistent with the variations by a factor of $\sim 1.8$ seen in the first 9 months of BAT observations [2]. While our analysis covers only time scales larger than a month, studies based on Suzaku investigated the variability on time scales of 
Table 1: Best-fit results for the INTEGRAL spectra during the different phases.

\begin{tabular}{ccccccccc}
\hline $\begin{array}{c}\text { Param } \\
\text { Phase }\end{array}$ & $\begin{array}{c}\chi^{2} / \\
\text { d.o.f. }\end{array}$ & $\Gamma$ & $\begin{array}{c}N_{\mathrm{H}}, 10^{23} \\
\mathrm{~cm}^{-2}\end{array}$ & $\begin{array}{c}E_{\mathrm{C}}, \\
\mathrm{keV}\end{array}$ & $\mathrm{HR}$ & $\mathrm{F}_{4-20}^{a}$ & $\mathrm{~F}_{20-60}^{a}$ & $\mathrm{~F}_{60-200}^{a}$ \\
\hline DSS & $9.7 / 11$ & $1.87 \pm 0.07$ & $6.4_{-1.8}^{+2.4}$ & - & $0.52_{-0.03}^{+0.02}$ & $0.45_{-0.05}^{+0.07}$ & $1.21_{-0.02}^{+0.03}$ & $1.58 \pm 0.04$ \\
DHS & $13.1 / 12$ & $1.24 \pm 0.12$ & $<7.9$ & $81_{-14}^{+19}$ & $0.08_{-0.03}^{+0.03}$ & $0.72_{-0.13}^{+0.12}$ & $1.14_{-0.04}^{+0.06}$ & $1.15_{-0.04}^{+0.05}$ \\
BHS & $13.9 / 13$ & $1.51 \pm 0.07$ & $3.5_{-1.0}^{+1.2}$ & $108_{-15}^{+20}$ & $0.04_{-0.05}^{+0.06}$ & $1.01_{-0.09}^{+0.09}$ & $1.87 \pm 0.05$ & $1.76 \pm 0.05$ \\
\hline
\end{tabular}

${ }^{a}$ Unabsorbed power-law flux given in $10^{-10} \mathrm{erg} /\left(\mathrm{cm}^{2} * \mathrm{sec}\right)$

Table 2: Seyfert galaxies with variable $X$-ray to $\gamma$-ray spectral shape.

\begin{tabular}{ccccc}
\hline Source & type & flux/slope & cut-off/min.value & reference \\
\hline IC 4329A & RQ S1 & uncorrelated & variable/40 keV & {$[22]$} \\
NGC 5548 & RQ S1 & correlated & variable/115 keV & {$[17]$} \\
NGC 3783 & RQ S1 & correlated & absent & {$[5]$} \\
NGC 4051 & RQ S1 & correlated & absent & {$[13]$} \\
NGC 4151 & RQ S1.5 & anticorrelated & variable/260 keV & {$[14]$} \\
NGC 2110 & RL S2 & uncorrelated & constant $/ 80 \mathrm{keV}$ & {$[6]$} \\
NGC 5506 & RQ S2 & uncorrelated & absent & {$[16]$} \\
NGC 4388 & RQ S2 & uncorrelated & variable/80 keV & this work \\
\hline
\end{tabular}

hours [8]. The variations at energies $>20 \mathrm{keV}$, where Compton thin absorbers have little influence on the observed flux, cannot be explained by the changes in the intrinsic absorbtion level.

In NGC 4388 we observe three different phases. The flux variations and spectral changes (i.e. of spectral slope and high-energy cut-off) are independent. The DHS cut-off and photon index are consistent within the errors with the values obtained by Beckmann et al. [3] based on the first $870 \mathrm{ks}$ of INTEGRAL/ISGRI observations (i.e. $E_{\mathrm{C}}=80 \mathrm{keV}$ and $\Gamma=1.3$ ). The high-energy cut-off of NGC 4388 as measured in recent $S u z a k u$ data [21] appears to be even lower, with $E_{\mathrm{C}}=30 \mathrm{keV}$ and an even harder spectral slope of $\Gamma=0.9$.

Several other AGNs with variable spectral shape are known. To compare their properties we summarize some parameters in Table 2. Three of them (NGC 3783, NGC 4051, NGC 5548) follow the pattern predicted by Poutanen \& Svensson [19]. The behaviour of NGC 4151 was interpreted as a result of temperature and optical depth changes in the accreting medium [14]. NGC 2110 and NGC 5506 demonstrate the behaviour more similar to NGC 4388 (i.e. no link between flux level and spectral shape). To explain such variations we suggest a more complex situation, for instance that both, density and temperature of the plasma in the corona change and also the disc/corona geometry is variable, as in the scenarios by Uttley et al. [23] and Petrucci et al. [18].

Another interesting question concerns the cut-off at energies lower than $100 \mathrm{keV}$, as seen in NGC 4388 and IC 4329A. In AGNs this is supposed to be connected to radio-loudness and energy losses in the jets or other types of outflows [12]. But NGC 4388 is a radio quiet object [9], and only a faint flat-spectrum radio source was detected by the VLBI [10]. This brings up a question about the radio emission of this Seyfert 2 core - is it permanently weak or are there also periods of high radio luminosity? Is the radio luminosity of this object connected to the flux and the spectral 
properties in the X-ray band? Thus it would be interesting to test this link, to observe NGC 4388 simultaneously in hard X-rays and at radio frequencies in order to trace out the link between the radio luminosity and spectral shape in $\mathrm{X}$-rays/ $\gamma$-rays.

\section{Acknowledgments}

This research has made use of data obtained through the HEASARC Online Service, provided by NASA/GSFC. EVF is grateful to V. Bianchin and L. Foschini for their help with the INTEGRAL software and to Kiev VIRGO.UA center for offering informational and technical facilities.

\section{References}

[1] V. Beckmann et al. INTEGRAL and XMM-Newton Spectral Studies of NGC 4388, ApJ 04 (614)641.

[2] V. Beckmann et al. Hard X-ray variability of active galactic nuclei, A\&A 07 (475)827.

[3] V. Beckmann et al. The second INTEGRAL AGN catalogue, A\&A 09 (505)417.

[4] T.J.-L. Courvoisier et al. Simultaneous observations of the quasar 3C 273..., A\&A 03 (411)L343.

[5] A. De Rosa et al. The BeppoSAX broad-band spectrum..., A\&A 02 (387) 838.

[6] V. Beckmann \& O. Do Cao, The elusive radio loud Seyfert 2 galaxy NGC 2110, these proceedings 11.

[7] M. Elvis et al. An Unveiling Event in the Type 2 AGN NGC 4388..., ApJ 04(615)L25.

[8] Y. Fukazawa et al. Suzaku Observations of the Seyfert 2 Galaxy NGC 4388, ASPC (373)165.

[9] J. F. Gallimore et al. A Survey of Kiloparsec-Scale Radio Outflows in RQ AGN, AJ 06 (132)546.

[10] M. Giroletti, F. Panessa The Faintest Seyfert Radio Cores revealed by VLBI, AJ 09(706)L260.

[11] C. G. Hanson et al., Coded mask X-ray images of the Virgo cluster. I - Hard X-rays from the Seyfert galaxy NGC 4388, MNRAS 90(242)262.

[12] L. C. Ho, "Low-State" Black Hole Accretion in Nearby Galaxies, Astroph.\& Sp.Sci. 05 (300)219.

[13] G. Lamer et al. X-ray spectral variability of the Seyfert galaxy NGC 4051, MNRAS 03 (338)323.

[14] P. Lubiński et al. Extreme flux states of NGC 4151 observed with INTEGRAL, MNRAS 10 (408)1851.

[15] P. Magdziarz \& A. A. Zdziarski, Angle-dependent Compton reflection of X-rays and $\gamma$-rays, MNRAS 95 (273) 837.

[16] R. K. Manchanda, High energy X-ray observations of NGC 5506, Adv.Sp.Res. 06 (38)1387.

[17] F. Nicastro, A Long Observation of NGC 5548 by BeppoSAX..., ApJ 00(536)718.

[18] P. O. Petrucci et al. Physical interpretation of the NGC 7469 UV/X-ray variability, A\&A 04(413)477.

[19] J. Poutanen \& R. Svensson, The Two-Phase Pair Corona Model for AGN..., ApJ 96 (470) 249.

[20] G. Risaliti, The BeppoSAX view of bright Compton-thin Seyfert 2 galaxies, A\&A 02 (386)379.

[21] H. Shirai et al. Detailed Hard X-Ray Measurements..., PASJ 08(60)263.

[22] S. Soldi et al. AGN variability at hard X-rays [astro-ph/1001 .4348].

[23] P. Uttley et al. Correlated Long-Term Optical and X-Ray Variations in NGC 5548, ApJ 03(584)L53. 